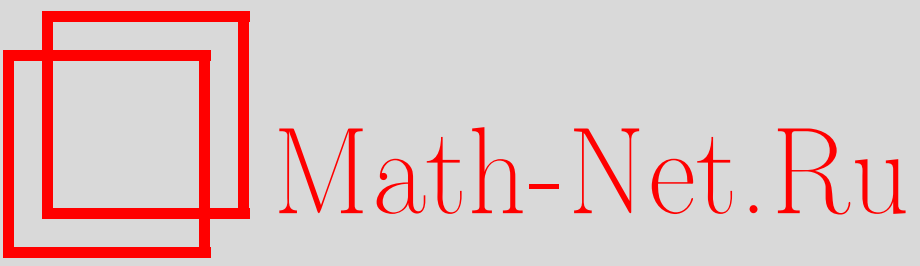

Н. С. Бахвалов, М. И. Зеликин, А. С. Калашников, В. Л. Камынин, О. А. Олейник, Е. Ю. Панов, Н. С. Петросян, В. М. Тихомиров, А. В. Фаминский, В. Н. Чубариков, Станислав Николаевич Кружков (некролог), УМН, 1998, том 53, выпуск 5, 213-220

DOI: https://doi.org/10.4213/rm73

Использование Общероссийского математического портала Math-Net.Ru подразумевает, что вы прочитали и согласны с пользовательским соглашением

http: //www.mathnet.ru/rus/agreement

Параметры загрузки:

IP : 107.22 .136 .117

26 апреля 2023 г., 16:02:59 


\section{СТАНИСЛАВ НИКОЛАЕВИЧ КРУЖКОВ}

12 июня 1997 года скоропостижно скончался доктор физико-математических наук профессор Кружков Станислав Николаевич.

С.Н. Кружков родился 16 сентября 1936 года в Москве. Он рано проявил интерес и способности к занятиям математикой: в возрасте 12 лет с увлечением читал книги из математической библиотеки своего отца, студента Московского университета; позже успешно проводил уроки у себя в школе вместо заболевшего учителя. Будучи старшеклассником, посещал школнньй кружок под руководством Н. С. Бахвалова при механико- математическом факультете МГУ. С Московским университетом неразрывно связана вся дальнейшая жизнь Станислава Николаевича. В 1954 году он становится студентом механико-математического факультета МГУ, в 1959 году оканчивает его и поступает в аспирантуру к О.А. Олейник. Обучаясь в аспиранту-

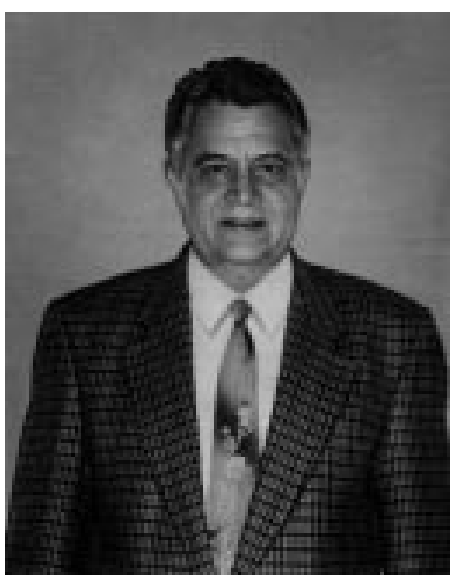
pe, начинает вести упражнения в студенческих группах.

Затем продолжает преподавание в качестве штатного сотрудника механико-математического факулттета МГУ, занимая последователшно должности ассистента, доцента, а с 1973 года и до конца своей жизни - профессора кафедры дифференциальных уравнений.

С.Н. Кружковым опубликовано более 100 научных работ, среди которых монографияя в двух частях [32], [33]. Станислав Николаевич подготовил 17 кандидатов физико-математических наук.

Полученные С. Н. Кружковым научные резултаты пользуются широкой известностью в нашей стране и за рубежом. Болшшинство их относится к теории нелинейных дифференциальных уравнений с частными производными.

В своей дипломной работе [1], выполненной под руководством О.А. Олейник, Станислав Николаевич ввел понятие глобального обобщенного решения (кратко: г.о.р.) задачи Коши для уравнения Гамильтона-Якоби $u_{t}+f\left(u_{x}\right)=0$ с гладкой выпуклой функцией $f$. Он доказал теоремы существования и единственности, получил явную формулу для г.о.р. и установил неожиданньй факт стабилизации г.o.p. при $t \rightarrow+\infty$ к нижней грани началшной функции, которьй впоследствии был интерпретирован как принцип "асимптотически наименьшего действия".

В статьях [4], [5], [6], опубликованных в 1961-1963 гг., дано развитие предложенной Мозером методики вывода априорных оценок для решений линейных и квазилинейных эллиптических и параболических уравнений второго порядка. С помощью полученных оценок доказана разрешимость основных краевых задач для таких уравнений и изучены свойства их решений. Эти результаты С. Н. Кружкова составили содержание его кандидатской диссертации "Априорные оценки решений и краевые задачи для эллиптических и параболических уравнений второго порядка", защищенной в 1963 г. 
Исследования, выполненные Станиславом Николаевичем в 1964-1970 гг., группируются преимущественно по трем направлениям.

В [20], [27] С. Н. Кружков с помощью предложенных им новых подходов доказал глобальную разрешимость основных краевых задач и задачи Коши для нелинейных параболических уравнений и систем произвольного порядка с двумя независимыми переменными. Эти результаты впоследствии получили дальнейшее развитие в [50], где рассмотрены также некоторые случаи вьрождения.

Работы [10], [12], [17], [18], [23] посвящены г.о.р. полностью нелинейных уравнений первого порядка с любым числом независимых переменных

$$
u_{t}+f\left(t, x, u, \nabla_{x} u\right)=0, \quad x=\left(x_{1}, \ldots, x_{n}\right) .
$$

В случае, когда функция $f$ вьпукла по последнему (векторному) аргументу, удалось распространить на такие уравнения описанные выше результаты из [1], а также дополнить их и интерпретировать в терминах теории оптимального управления. В [29], [30] рассмотрены уравнения вида (1) с невыпуклой $f$, возникающие в теории дифференциальных игр: получены минимаксно-максиминные представления локальных классических решений задачи Коши; указаны условия, при которых минимакс или максимин дает г.о.р. (цену соответствующей дифференциальной игры).

Одним из наиболее значителных научных достижений Станислава Николаевича является построенная им в конце 60 -х гг. теория г.о.р. многомерных квазилинейных уравнений первого порядка

$$
u_{t}+\sum_{i=1}^{n} \frac{d}{d x_{i}} \varphi_{i}(t, x, u)+\psi(t, x, u)=0, \quad x=\left(x_{1}, \ldots, x_{n}\right),
$$

без предположений о вьпуклости функций $\varphi_{i}$. Интерес к этой тематике стимулируется ее важностью для приложений: в частности, уравнения вида (2) можно рассматривать как модельные для систем, возникающих в газовой динамике. В случае, когда $n=1$ и $\varphi_{1}$ uи $>0$, теория г.o.p. ранее была построена О.А. Олейник. В общем случае С.Н. Кружков предложил определение г.о.р. задачи Коши для уравнения (2), опирающееся на интегралнне неравенство, которое содержит (наряду с пробной функцией) числовой параметр. Следствиями этого неравенства для г.о.р. являются как выполнение (2) в слабом смысле, так и справедливость соотношения на линиях разрыва, моделирующего налагаемое в газовой динамике требование возрастания энтропии на ударных волнах. Для введенных г.о.р. С.Н. Кружков доказал [26], [28], [34] теоремы существования, единственности, сравнения и непрерывной зависимости от начальных данных. При этом были развиты новые методы исследования: например, для доказателшства единственности применено удвоение числа независимых переменных.

Работы Станислава Николаевича, посвященные квазилинейным уравнениям первого порядка, получили всемирную известность. Предложенное им определение г.о.р. вошло в число фундаменталшных понятий теории нелинейных уравнений с частными производными как "энтропийное решение в смысле Кружкова". Это определение наряду с обобщением на случай систем, также сфформулированным в [34], нашло многочисленные применения. Так, на основе этого понятия удалось вьвести оценки для погрешности численного интегрирования квазилинейного уравнения первого порядка в общем случае, что́ ранее считалось невозможным при отсутствии предположений типа выпуклости. На нескольких международных конференциях по теоретическим проблемам численных методов газовой динамики С.Н. Кружков был одним из наиболее часто цитируемых авторов.

Цикл работ, выполненных Станиславом Николаевичем в 1964-1970 гг., послужил основой его докторской диссертации "K нелокальной теории краевых задач и задачи Коши для нелинейных уравнений с частными производными", защищенной в 1970 г.

В 70-х гг. С. Н. Кружковым была исследована [41], [42] краевая задача, названная им задачей Коши-Дирихле, для "стационарных" полностью нелинейных уравнений первого порядка, типичным представителем которых может служить возникающее в геометрической оптике уравнение 
эйконала $|\nabla u(x)|^{2}=1$. Было введено понятие г.о.р. этой задачи, доказаны его существование, единственность и непрерьвная зависимость от граничных данных, обоснованы обобщенные принципы Ферма и Гюйгенса.

В середине 70-х гг. Станислав Николаевич совместно со своими учениками начал [38], [39] построение теории г.о.р. задачи Коши для квазилинейных уравнений первого порядка, которым соответствует бесконечная скорость распространения возмущений; такой эффект может возникать, например, если в уравнении (2) функции $\varphi_{i}$ не обладают ограниченными производными по $u$. Затем эти исследования были продолжены в [76], [81], [85], [96], [97], [105], [106]. Было доказано, что существование г.о.р. имеет место без каких-либо предположений о модулях непрерывности функций $\varphi_{i}$. Был найден ряд достаточных условий единственности, формулируемых в терминах этих модулей непрерывности; показано, что налагаемые условия близки к необходимым; изучены свойства г.о.р., в частности установлена устойчивость нулевого решения в нормах пространств $L_{p}$.

В 80-х и 90-х гг. С. Н. Кружков совместно со своими учениками получил болшшое число интересных результатов, относящихся к различным разделам теории нелинейных уравнений с частными производными. В [56], [57], [58], [72] изучалось поведение г.о.р. квазилинейных уравнений первого порядка при больших значениях времени. В [55], [62] исследовались г.о.р. задачи Коши для уравнения Кортевега-де Фриза с нерегулярньми начальными данными. В связи с задачами механики композитов рассматривался вопрос о сходимости решений квазилинейных параболических уравнений при наличии слабой сходимости их коэффициентов [59], [61], [69].

Несколко работ С. Н. Кружкова содержат решение конкретных прикладных задач. Еще будучи студентом, он совместно с профессором Московского института неф̆ти и газа Б. Б. Лапуком рассчитал предельные безводные (а следовательно, безаварийные) дебиты газовых скважин в залежах с подошвенной водой; полученные формулы были использованы на практике. Математическому моделированию процессов двухфазной фильтрации (например, в случае двухкомпонентной смеси нефти с водой) посвящены статьи [46], [47], [68]; в них доказаны теоремы о классической и обобщенной разрешимости соответствующих краевых задач, предложена конструкция приближенных решений и даны оценки скорости их сходимости к точному решению. В работах [21], [25], [43], [49], [54], [65] исследована поставленная Г. И. Баренблаттом и А.Ю.Ишлинским задача об ударе вязкопластического стержня о жесткую преграду (моделирующая, в частности, столкновение поездов): доказана теорема единственности, построено эквивалентное функциональное уравнение и изучены качественные свойства его решения. В [84], [89] рассмотрены некоторые задачи химической кинетики.

Не имея возможности описать здесь все резултаты С. Н. Кружкова, относящиеся к уравнениям с частными производными, отметим еще несколько его работ на другие темы. В [44] pacсмотрен вопрос о дифференцируемости почти всюду функций нескольких переменных. В [63], $[66],[67]$ получены обобщения классических теорем о вложении функциональных пространств на анизотропный случай.

С.Н. Кружкова очень волновал вопрос о подготовке научной смены. Выезжая с лекциями в различные города, он занимался популяризацией математики, специально искал способных молодых людей и старался привлечь их к обучению в качестве студентов и аспирантов. В частности, он систематически вел такую работу в Новгороде.

Станислав Николаевич щедро делился своими идеями с многочисленными учениками. Они всегда могли рассчитывать на его помошь как в научной работе, так и при решении житейских проблем.

С. Н. Кружков много сделал для увековечения памяти великого русского математика П. Л. Чебышёва. По инициативе Станислава Николаевича на механико-математическом факултете были основаны регулярные "Чебышёвские чтения", проведена международная конференция, посвященная 175-летию со дня рождения П. Л. Чебышёва, организовано шефбство факулттета над школой имени П. Л. Чебышёва в Калужской области.

Станислав Николаевич был человек с разносторонними интересами: автомобилист, фотолюбитель, турист, яхтсмен, рыболов, грибник. Но на первом месте для него всегда стояла мате- 
матика, увлеченность которой не покидала его до самой кончины. Имя его навеки сохранится в истории нашей науки.

Н. С. Бахвалов, М.И. Зеликин, А.С. Калашников, В. Л. Камынин, О. А. Олейник, Е. Ю. Панов, Н. С. Петросян, B. М. Тихомиров, А.В. Фаминский, В.Н. Чубариков

\section{СПИСОК НАУЧНЫХ РАБОТ С. Н. КРУЖКОВА ${ }^{1}$}

[24] Краевые задачи для вырождаюшихся эллиптических уравнений второго порядка // Матем. сб. 1968. Т. 77. № 3. С. 288-334

[25] Об одном классе задач с неизвестной границей для уравнения теплопроводности // Докл. AH CCCP. 1968. T. 178. № 5. C. 1036-1038

[26] Обобщенные решения задачи Коши в целом для нелинейных уравнений первого порядка // Докл. АН СССР. 1969. Т. 187. №1. С. 29-32

[27] Линейные и нелинейные параболические системы на плоскости // Докл. АН СССР. 1969. T. 187. № 3. C. $510-513$

[28] Резултаты о характере непрерьвности решений параболических уравнений и некоторые их применения // Матем. заметки. 1969. Т. 6. № 1. С. 97-108

[29] Нелинейные уравнения первого порядка и связанные с ними дифференциальные игры // УМH. 1969. Т. 24. № 2. С. 227-228

[30] О минимаксном представлении решений нелинейных уравнений первого порядка // Функцион. анализ и его приложения. 1969. Т. 3. № 2. С. 57-66

[31] О задаче Коши для некоторых классов квазилинейных параболических уравнений // Матем. заметки. 1969. Т. 6. №3. С. 295-300

[32] Нелинейные уравнения с частными производными. Часть 1. Эллиптические и параболические уравнения второго порядка. М.: Изд-во МГУ, 1969

[33] Нелинейные уравнения с частными производными. Часть 2. Уравнения первого порядка. М.: Изд-во МГУ, 1970

[34] Квазилинейные уравнения первого порядка со многими независимыми переменными // Матем. сб. 1970. Т. 81. № 2. С. 228-255

[35] Периодические решения нелинейных параболических уравнений второго порядка // Дифоференц. уравнения. 1970. Т. 6. № 4. С. 731-740

[36] Об основной априорной оценке для решений квазилинейного параболического уравнения // Изв. АН УзССР. Сер. физ.-матем. наук. 1972. № 3. С. 16-20

[37] Априорные оценки и неравенство Харнака для обобщенных решений вырождающихся квазилинейных параболических уравнений второго порядка // Докл. АН СССР. 1972. Т. 204. № 2. С. 283-284 (совм. с Колодием И. М.)

[38] Задача Коши для квазилинейных уравнений первого порядка в случае, когда область зависимости от началных данных бесконечна // Вестн. МГУ. Сер. матем., мех. 1974. № 1. С. 93-100 (совм. с Хильдебрандом $\Phi$.

[39] К нелокальной теории задачи Коши для квазилинейных уравнений первого порядка в классе локально суммируемшіх функций // Докл. АН СССР. 1975. Т. 220. № 1. С. 23-26 (совм. с Андреяновым П. А.)

[40] Оценки шаудеровского типа и теоремы существования решений основных краевых задач для линейных и нелинейных параболических уравнений // Докл. АН СССР. 1975. Т. 220. № 2. С. 277-280 (совм. с Кастро А. и Лопесом М.)

[41] Задача Коши-Дирихле для уравнений Гамильтона-Якоби типа эйконала // Докл. АН CCCP. 1975. T. 224. № 5. С. 1012-1015

\footnotetext{
${ }^{1}$ Начало списка см. в кн.: Математика в СССР. 1958-1967. Т. 2. Вьп. 1. М.: Наука, 1969. C. 693 .
} 
[42] Обобщенные решения уравнений Гамильтона-Якоби типа эйконала. І. Постановка задач, теоремы существования, единственности и устойчивости, некоторые свойства решений // Матем. сб. 1975. Т. 98. № 3. С. 450-493

[43] О некоторых вопросах качественной теории параболических уравнений, связанных с задачей об ударе стержня // Динамика сплошной среды. 1975. № 23. С. 111-131

[44] К вопросу дифференцируемости почти всюду фуунций многих переменных // Вестн. МГУ. Сер. матем., мех. 1976. №6. С. 67-70

[45] Априорные оценки и неравенство Харнака для обобщенных решений вырождающихся квазилинейных параболических уравнений // Сиб. матем. журн. 1977. Т. 18. № 3. С. 608-628 (совм. с Колодием И.М.)

[46] Краевые задачи для систем типа двухфазной фильтрации: постановка задач, вопросы разрешимости, обоснование приближенных методов // Матем. сб. 1977. Т. 104. № 1. С. 69-88 (совм. с Сукорянским С.М.)

[47] О некоторых вопросах теории нелинейных дифференциальных уравнений, связанных с задачами филштрации // УМН. 1978. Т. 33. №3. С. 148-149

[48] Нелокальная теория уравнений Гамильтоа-Якоби // Труды Всес. конфер. по уравнениям с частными производными, посвящ. 75-летию со дня рождения акад. И. Г. Петровского. М.: Изд-во МГУ, 1978. С. 137-140

[49] О разрешимости одного класса задач с неизвестной границей для уравнения теплопроводности и поведении решений при неограниченном возрастании времени // Динамика сплошной среды. 1978. № 36. С. 46-70 (совм. с Якубовым С.)

[50] Квазилинейные параболические уравнения и системы с двумя независимыми переменными // Труды семинара им. И. Г. Петровского. 1979. № 5. С. 217-272

[51] Об анизотропных оценках шаудеровского типа для решений эллиптических уравнений // Докл. АН СССР. 1980. Т. 254. № 4. С. 788-791 (совм. с Лопесом М.)

[52] Об оценках по малой вязкости в некоторых нелинейных задачах // УМН. 1980. Т. 35 . № 4. C. 144

[53] Mayoraciones de Schauder y teoremas de existencia de las soluciones del problema de Cauchy para ecuaciones parabolicas lineales y no lineales. I / Ciencias Matematicas (Univ. Habana, Cuba). V. 1. № 1, 1980. P. 55-77 (with Castro A., Lopez M.)

[54] Задачи с неизвестной границей для параболических уравнений // УМН. 1981. Т. 36 . № 4. C. 200

[55] Обобщенные решения уравнения Кортевега-де Фриза // Докл. АН СССР. 1981. Т. 261. №6. С. 1296-1298 (совм. с Фаминским А. В.)

[56] Асимптотика по времени $t \rightarrow+\infty$ обобщенных решений задачи Коши для нелинейных уравнений первого порядка // Динамика сплошной среды. 1982. № 54. С. 100-114

[57] Об асимптотических свойствах решений некоторых классов нелинейных задач // Труды VII Советско-чехословацкого семинара "Применение методов теории функций и функционального анализа к задачам математической физики”. Ереван, 1982. С. 181-186

[58] Асимптотика решений задачи Коши для квазилинейных уравнений первого порядка // Докл. АН СССР. 1982. Т. 265. № 4. С. 801-804 (совм. с Петросян Н. С.)

[59] О сходимости решений параболических уравнений со слабо сходяшимися коэффициентами // УМН. 1982. Т. 37. № 4. С. 134 (совм. с Камыниным В. Л.)

[60] Mayoraciones de Schauder y teoremas de existencia de las soluciones del problema de Cauchy para ecuaciones parabolicas lineales y no lineales. II / Ciencias Matematicas (Univ. Habana, Cuba). V. 3. № 1, 1982. P. 37-57 (with Castro A., Lopez M.)

[61] Сходимость решений квазилинейных параболических уравнений со слабо сходящимися коэффициентами // Докл. АН СССР. 1983. Т. 270. № 3. С. 533-536 (совм. с Камыниным В. Л.)

[62] Обобщенные решения задачи Коши для уравнения Кортевега-де Фриза / / Матем. сб. 1983. Т. 120 (162). № 3. С. 396-425 (совм. с Фаминским А. В.)

[63] К теоремам вложения анизотропных пространств Соболева // УМН. 1983. Т. 38 . № 2. С. 207-208 (совм. с Колодием И. М.) 
[64] О свойствах непрерьвности решений некоторых классов нестационарных уравнений // Вестн. МГУ. Сер. матем., мех. 1983. № 3. С. 29-36 (совм. с Фаминским А. В.)

[65] Задача об ударе вязкопластического стержня и связанные с ней вопросы теории уравнения теплопроводности // Тезисы докл. Республиканской конфер. по нелинейным задачам матем. физики. Донецк: ИПММ АН УССР, 1983. С. 72

[66] Теоремы вложения анизотропных пространств и квазилинейные уравнения второго порядка // УМН. 1984. Т. 39. № 4. С. 134-135 (совм. с Королёвым А. Г.)

[67] К теории вложения анизотропных функциональных пространств // Докл. АН СССР. 1985. Т. 285. № 5. С. 1054-1057 (совм. с Королёвым А. Г.)

[68] Об единственности решений смешанных задач для вырождающейся системы двухфазной фильтрации // Вестн. МГУ. Сер. матем., мех. 1985. № 2. С. 28-32

[69] О предельном переходе в квазилинейных параболических уравнениях // Труды МИАН. 1985. Т. 167. С. 183-206 (совм. с Камыниным В. Л.)

[70] Фундаментальные решения задачи Коши для квазилинейных уравнений первого порядка // УМH. 1985. Т. 40. № 5. С. 192-193

[71] Развитие понятия обобщенного решения в теории нелинейных уравнений первого порядка // УМH. 1986. T. 41. № 5. C. 212-214

[72] Стабилизация решений некоторых квазилинейных параболических систем // УМН. 1987. Т. 42. № 4. С. 119 (совм. с Перегудовым А.Н.)

[73] Асимптотическое поведение решений задачи Коши для нелинейных уравнений первого порядка // УМН. 1987. Т. 42. № 5. С. 3-40 (совм. с Петросян Н. С.)

[74] Разрьвные решения некоторых существенно нелинейных уравнений первого порядка // УМН. 1988. Т. 43. № 4. С. 182

[75] Некоторые нерешенные задачи теории дифференциальных уравнений и математической физики // УМН. 1989. Т. 44. № 4. С. 191-202 (совм. с Арнолдомм В. И., Вишиком М. И., Ильяшенко Ю.С., Калашниковым А. С., Кондратьевым В.А., Ландисом Е. М., Миллионщиковым В. М., Олейник О. А., Филипповым А. Ф., Шубиным М.А.)

[76] Консервативные законы с бесконечной областью зависимости от начальных данных // УМН. 1989. Т. 44. № 4. С. 222-223 (совм. с Пановым Е. Ю.)

[77] Об одной математической модели фазового перехода // Тезисы докладов Всес. конфб. "Новые подходы к решению дифференциальных уравнений”. М.: ВЦ АН СССР, 1989

[78] О некоторых примерах неединственности решения задачи Коши для квазилинейных гиперболических систем // Вестн. МГУ. Сер. матем., мех. 1990. № 5. С. 80-84

[79] К методам построения обобщенных решений квазилинейных законов сохранения // УМН. 1990. Т. 45. № 4. С. 111-112 (совм. с Казмерчуком А. И.)

[80] Некоторые вопросы нелокальной теории нелинейных уравнений // Тезисы докладов III Всес. школы "Оптимальное управление, геометрия и анализ" ("Понтрягинские чтения"). Кемерово: 1990. С. 37

[81] Консервативные квазилинейные законы первого порядка с бесконечной областью зависимости от начальных данных // Докл. АН СССР. 1990. Т. 314. № 1. С. 79-84 (совм. с Пановым Е. Ю.)

[82] Задача Коши для квазилинейных законов сохранения // Тезисы докладов III Междунар. конф. “Лаврентьевские чтения по математике, механике и физике”. Новосибирск, 1990. C. $32-33$

[83] Обобщенные решения нелинейных уравнений первого порядка // Нелинейные граничные задачи. Вып. 2. Киев: Наукова думка, 1990. С. 56-60

[84] Задача Коши для системы квазилинейных параболических уравнений типа химической кинетики: разрешимость и стабилизация решений при $t \rightarrow+\infty / /$ Препринт Ин-та структурной макрокинетики АН СССР (г. Черноголовка), 1990. С. 1-29 (совм. с Перегудовым А. Н.)

[85] Conservation laws with infinite domain of dependence on initial data // Differential Equations and Control Theory (Pitman Research Notes in Mathematics. V. 250). Harlow: Longman, 1991. P. 149-162 
[86] О теории обобщенных глобальных решений нелинейных уравнений первого порядка // УМН. 1991. Т. 46. №6. С. 172-173

[87] К 170-летию со дня рождения П. Л. Чебышёва // Вестн. МГУ. Сер. матем., мех. 1991. № 5. C. $2-4$

[88] Теория функций и диффференциальные уравнения // Вестн. МГУ. Сер. матем., мех. 1991. № 5. C. $36-43$

[89] Задача Коши для системы квазилинейных параболических уравнений типа химической кинетики // Труды семинара им. И. Г. Петровского. 1992. № 16. С. 242-261 (совм. с Перегудовым А.Н.)

[90] Нелокальная теория нелинейных уравнений первого порядка // XVII Национална лятна школа (Варна, 1991). Сборник доклади и научни съобщения. София: Изд-во Софийского технического ун-та, 1992. С. 43-63

[91] Нелинейные параболические уравнения второго порядкана плоскости // УМН. 1993. Т. 48. № 4. С. 186 (совм. с Барсовым О.Н.)

[92] Краевые задачи и задача Коши для нелинейных параболических уравнений на плоскости // XVIII Национална лятна школа (Варна, 1992). Сборник доклади и научни съобщения. София: Изд-во Софийского технического ун-та, 1993. С. 1

[93] First-order conservation laws with continuous flux function // Abstracts of Communications, Seminarios Departamento de Matematicas, Universidad Autonoma de Madrid, Seminario de EDP e Matematica Aplicada, Programa Triple, 4.02.1994. P. 1

[94] Global generalized solutions to first order conservation laws with anisotropic continuous flux functions // Abstracts of Short Communications, International Congress of Mathematicians ICM-94, Zürich, August 1994. P. 172

[95] Задача Коши для некоторых моделшных квазилинейных законов сохранения // УМН. 1994. Т. 49. № 4. С. 82-83

[96] Квазилинейные уравнения первого порядка с непрерьвными нелинейностями / / Докл. АН. 1994. Т. 339. № 2. С. 151-154 (совм. с Бениланом Ф.)

[97] Conservation laws with continuous flux functions // Prepublications de l'Equipe de Mathematiques de Besançon. Besançon: Université de Franche Compte, 1994. № 94/23. P. 1-25 (with Benilan Ph.)

[98] Osgood's type conditions for uniqueness of entropy solutions to Cauchy problem for quasilinear conservation laws of the first order // Annali Univ. Ferrara. Ser. VII. Sci. Mat. 1994-1995. V. 40. P. 31-53 (with Panov E. Yu.)

[99] Условия типа Осгуда в проблеме единственности обобщенного энтропийного решения задачи Коши для квазилинейных законов сохранения первого порядка при наличии эффекта бесконечной скорости распространения возмущений // УМН. 1995. Т. 50. № 4. С. 77 (совм. с Пановым Е. Ю.)

[100] Effect of infinite speed of propagation in the nonlocal theory of conservation laws // Invited Speakers' Abstracts of International Conference EQUADIFF-95. Lisbon, 1995. P. 3

[101] Uniqueness problem in $L_{1}$ for scalar conservation laws with infinite speed of propagation // Book of Abstracts of the International Conference "Nonlinear Differential Equations". Kiev, 1995. P. 17 (with Benilan Ph.)

[102] Uniqueness and regularity of entropy solutions to Cauchy problem for quasilinear first order equations // Book of Abstracts of the International Conference "Nonlinear Differential Equations". Kiev, 1995. P. 89 (with Panov E. Yu.)

[103] К 100-летию со дня смерти Пафнутия Львовича Чебьшёва // Вестн. МГУ. Сер. матем., мех. 1995. № 6. С. 3-12

[104] Нелинейные параболические уравнения второго порядка на плоскости // Вестн. МГУ. Сер. матем., мех. 1995. № 6. С. 21 (совм. с Барсовым О. Н.)

[105] On some properties of generalized entropy solutions to first order conservation laws with infinite speed of propagation // FBP News. 1996. № 10. P. 7-8 
[106] Conservation laws with continuous flux functions // NoDEA. 1996. V. 3. P. 395-419 (with Benilan Ph.)

[107] Propagation properties for scalar conservation laws // Comptes Rendus Acad. Sci. Paris. Ser. 1. 1996. V. 323. № 5. P. 463-468 (with Dias Kh. I.)

[108] Условия типа Осгуда в проблеме единственности обобщенного энтропийного решения задачи Коши для квазилинейных уравнений первого порядка // Вестник РУДН. Математика. 1996. Т. 3. №1. С. $72-91$ (совм. с Пановьд Е.Ю.)

[109] К 175-летию со дня рождения Паффнутия Львовича Чебышёва // Вестн. МГУ. Сер. матем., мех. 1996. № 6. С. $3-4$

[110] Квазилинейные уравнения с частньми производными первого порядка: обобщенные решения, ударные волны, центрированные волны разрежения (Краткое учебное пособие). М.: Изд-во механико-математического факултета МГУ, 1997 (совм. с Горицким А. Ю., Чечкиным Г.А.) 